\title{
Die EU nach Lissabon - bürgernah, bürgerfreundlich, bürgertauglich?
}

\author{
Melanie Piepenschneider*
}

\section{Europa und die Bürger - gegenseitige Verantwortung für die europäische Einigung}

Das Jahr 2009 ist durch eine ganze Reihe von Jubiläen gekennzeichnet: So jährt sich zum 70. Mal der Beginn des Europa zerstörenden Zweiten Weltkrieges. Vor 60 Jahren wurde der Europarat gegründet. Das Europäische Parlament wird zum siebten Mal und seit 30 Jahren direkt von den Bürgerinnen und Bürgern gewählt. Europa feiert das 20-jährige Jubiläum der Überwindung des Ost-West-Konflikts und des Falls der Mauer. Polen, Ungarn und Tschechien begehen ihre zehnjährige Mitgliedschaft in der NATO.

Diese wenigen Daten skizzieren die Prägezeichen des letzten Jahrhunderts und geben einen Hinweis auf kommende Herausforderungen: Ein von Kriegen nicht nur materiell vernichtetes Europa versucht in einer enormen Kraftanstrengung die geschichtlich immer wiederkehrende Abfolge von Feindschaft, Krieg, Zerstörung und knappen Phasen des Friedens zu durchbrechen. Krieg sollte zwischen den Staaten Europas nicht mehr möglich sein. Hierzu bedurfte es organisatorischer Strukturen und institutioneller Regeln zur Gestaltung gemeinsamen Handelns und zum Einhegen von Verteilungskämpfen über Ressourcen: Diese Motive waren es unter anderem, die vor 60 Jahren, am 5. Mai 1949, zur Gründung des Europarates führten. Die noch nach Spielregeln klassischer internationaler Zusammenschlüsse verfasste Organisation Europarat war der erste Versuch der Vertragspartner gemeinsam politische Herausforderungen zu bewältigen. Durch die Schaffung der Europäischen Gemeinschaft für Kohle und Stahl Anfang der Fünfzigerjahre wurde dann das ehrgeizige europäische Integrationsprojekt begonnen.

Die europäische Einigung hatte immer das Ziel, das friedliche Zusammenleben unterschiedlicher Völker zum Wohle aller zu organisieren. Dies schloss eine friedliche und damit kriegsfreie Existenz genauso ein wie die Schaffung und Sicherstellung von Freiheit und Wohlstand. Dieses Friedensprojekt ist eine unvergleichliche europäische Erfolgsgeschichte. Nach den Erfahrungen zweier Weltkriege im 20. Jahrhundert war der Boden bereitet, den friedlichen Weg über vertragliche Vereinbarungen für gemeinsam zu lösende Fragen und Probleme zu gehen.

Begonnen wurde das europäische Einigungswerk als ein Projekt der Eliten. Denn Paradigmenwechsel dieser Größenordnung kommen entweder durch (blutige) Revolutionen oder Kriege zustande oder Eliten müssen in einem Akt des Willens vorangehen. Die Europäische Union entwickelte sich im Laufe der Jahre aber von einem Europa für die Bürger zu einem Europa der Bürger. Diese Veränderung hat eine Neujustierung von Verantwortlichkeiten zur Folge:

- Die Bürger haben eine Verantwortung für Europa: So wie die Bürger Ost- und Mitteleuropas 1988/1989 und in der DDR in der Friedlichen Revolution die Verantwortung für Gesellschaft und Staat selbst in die Hand nahmen, indem sie sich von Diktaturen befreiten, genauso darf auch den Bürgern das Schicksal der europäischen Einigung nicht gleichgültig

* Dr. Melanie Piepenschneider, Leiterin Hauptabteilung Politische Bildung, Konrad-Adenauer-Stiftung, Wesseling (bei Köln). 
sein. Hierbei geht es nicht um eine erneute Revolution im Sinne des Einreißens von Mauern und Zäunen, aber um die Überwindung geistiger Barrieren und von Lethargie. Es geht um die Identifikation mit dem europäischen Integrationsprojekt und die Mobilisierung, sich für die Idee der Einigung Europas einzusetzen und bei dessen Ausgestaltung mitzuwirken.

- Vor 20 Jahren sind die Bürger in Europa und der DDR gegen Unfreiheit und Diktatur auf die Straße gegangen: die Bürger Europas haben sich Freiheit und Demokratie erkämpft. Dadurch entsteht auf der anderen Seite auch eine Verpflichtung Europas gegenüber seinen Bürgern. Der Kampf für Freiheit und Demokratie darf nicht an den nationalen Grenzen Halt machen.

In dieser gegenseitigen Verpflichtung müssen die Anstrengungen betrachtet und bewertet werden, die europäische Einigung bürgernah, bürgerfreundlich und bürgertauglich zu machen. Denn ohne Bürger ist das europäische Integrationsprojekt obsolet - nur um seiner selbst Willen macht es wenig Sinn und ist zum Scheitern verurteilt. Strukturen neuer Machtausübung zu schaffen ohne Rückkopplung oder Verbindung zu den Bürgern wird auf Dauer keinen Bestand haben - es sei denn, diese Strukturen enden (wieder) in einer Diktatur; und selbst diese haben nur eine zeitlich begrenzte Lebensdauer wie die Geschichte lehrt.

\section{Die Europäische Union - bürgernah, bürgerfreundlich, bürgertauglich?}

Unbestreitbar hat die Europäische Union im Vergleich mit anderen Organisationen den höchsten Grad an Konkretisierung und Strukturierung europäischen politischen Handelns erreicht.

Solange die Europäische Union neben lokaler, regionaler und staatlicher Ebene ,nur ' eine weitere Bühne für politisch Handelnde im Interesse ihres Nationalstaates darstellte, wurde die Frage nach dem Beziehungsgeflecht zwischen Europäischer Union und Bürger vor allem in Fachkreisen gestellt und diskutiert. Die Europäische Union wurde im Laufe der Jahre eine ,Macht' an sich und war nicht mehr nur eine Struktur, über die Repräsentanten ihrer Mitgliedstaaten das eigene Machtpotenzial ausweiten konnten. Je mehr sich die Europäische Union zu eben diesem eigenen Akteur auf der Weltbühne entwickelte (und dies auch musste), je mehr Rechte und Kompetenzen die Organe der Europäischen Union durch Vertragsrevisionen erhielten, desto angespannter wurde das Verhältnis zwischen der Europäischen Union und ihren Bürgern. ${ }^{1}$

Ebenso kann man feststellen, dass je mehr Wissen über die Europäische Union beim Bürger vorhanden ist, er umso kritischer die Einigungsbemühungen beurteilt. Dies soll kein Plädoyer gegen politische Bildung oder Informationskampagnen der Europäischen Kommission oder anderer Stellen zum Thema Europa sein. Dieses Phänomen macht allerdings deutlich, dass eine intensive Informationspolitik nicht ,brave', angepasste und positiv gestimmte Europäer schafft, sondern sie einem hohen argumentativen Standard genügen muss und nicht ein Selbstläufer im Hinblick auf die Erhöhung von Zustimmungsraten in der Bevölkerung ist.

Zudem kann es passieren, dass durch eine immer größere Verdichtung des europäischen Integrationsgeflechts und des damit einhergehenden Transfers von mehr und mehr Rechten an eine übergeordnete Politikebene der Bürger das Empfinden hat, dass seine Freiheitsrechte begrenzt werden. Es muss im Zuge der fortschreitenden Integration Europas auch immer

1 Siehe auch Thomas Petersen: Der Kampf Europas mit der Gleichgültigkeit, in: Frankfurter Allgemeine Zeitung, 21.05.2008; Heinrich Neisser: Einstellungen der Bürger zur europäischen Einigung im Wandel, in: Otto Schmuck (Hrsg.): Die Menschen für Europa gewinnen - Für ein Europa der Bürger, Bad Marienberg 2008, S. 129-146. 
wieder die (Kontroll-)Frage gestellt werden, ob der Freiheitsgrad der Bürger durch die Europäische Union zugenommen hat oder ob die Bürger durch sie in ihrer (staatlich garantierten) Freiheit eher begrenzt werden? ${ }^{2}$

Das Ziel von politischem Handeln ist, Gesellschaft so zu organisieren, dass das ,größte Glück der größten Zahl“3 möglich wird. Dieses Bestreben treibt nicht nur Staaten an, sondern ist in Zeiten der Globalisierung längst nationalen Grenzen entwachsen. Die aktuelle Wirtschafts- und Finanzkrise verdeutlicht die (weltweite) Verflechtung im Bereich der Wirtschaft und zeigt, dass nur aufeinander abgestimmtes, gemeinsames politisches Handeln zu Lösungen für die europäischen Staaten führen kann. Damit bekommt die europäische Einigung einen den aktuellen Gegebenheiten angepassten und verdichteten Auftrag und unterstreicht das politische Projekt Europa.

In einem ersten Schritt gilt es, die nicht ganz spannungsfreie Wechselbeziehung zwischen Europäischer Union und dem Bürger zu definieren. Das Beziehungsgeflecht ist auf mehreren, unterschiedlichen Ebenen zu betrachten:

1. Wertegemeinschaft: Inwiefern verbinden die Unionsbürger gemeinsame Werte und wie haben diese Eingang in das europäische Vertragswerk gefunden?

2. Rechte und Pflichten: Inwiefern schützt die Europäische Union die Rechte der Bürger und definiert deren Pflichten gegenüber der Europäischen Union?

3. Demokratisierung: Inwiefern ist der Bürger als Akteur mittel- und unmittelbar am europäischen Integrationsprozess beteiligt beziehungsweise in diesen einbezogen?

4. Transparenz und Effizienz: Inwiefern sind die Strukturen und Entscheidungsprozesse in der Europäischen Union transparent und effizient und vermitteln dem Bürger einen Mehrwert oder Zugewinne an Sicherheit, Freiheit und Wohlfahrt?

Ausgangspunkt der Betrachtung ist der Vertrag von Lissabon: ${ }^{4}$ Auch wenn er noch nicht in Kraft getreten und damit kein geltendes Recht ist, wird ihm bisher doch der höchste Grad an Verwirklichung von Bürgernähe und Demokratie zugeschrieben. ${ }^{5}$ Diese Bewertung des Lissabonner Vertrages wird auch durch sein Zustandekommen gestützt: Vorläufer war der Entwurf eines Vertrags über eine Verfassung für Europa. ${ }^{6}$ Dieser Verfassungsvertragsentwurf wurde vom Konvent zur Zukunft Europas (Europäischer Konvent) ausgearbeitet. Der Konvent war auch deshalb beachtenswert, weil neben Mitgliedern der Regierungen, Abgeordnete des Europäischen Parlaments und der nationalen Parlamente vertreten waren. Somit waren vom Bürger gewählte Repräsentanten am Zustandekommen, an Inhalt und Formulierungen direkt beteiligt. Aber auch die Zivilgesellschaft wirkte durch zahlreiche Foren und andere Möglichkeiten der Artikulation von Standpunkten und Ideen auf den Entstehungsprozess des Textes ein. ${ }^{7}$

2 Diese Fragestellung ist unter anderem auch eine der Argumentationen beziehungsweise Begründungen für Klagen gegen den Lissabonner Vertrag vor dem Bundesverfassungsgericht.

3 Abgewandeltes Zitat von Francis Hutcheson (1694-1746), irischer Philosoph und Ökonom: ,größte Beglückung für die größte Anzahl“.

4 Die gemäß dem Lissabonner Vertrag konsolidierten Fassungen des Vertrags über die Europäische Union (EUV) und des Vertrags über die Arbeitsweise der Europäischen Union (AEUV) sind abgedruckt in: Amtsblatt der EU, Nr. C 115 vom 9. Mai 2008.

5 Eine Auszählung der Begriffe Bürger/innen und Unionsbürgerschaft in den konsolidierten Fassungen des Vertrags über die Europäische Union und des Vertrags zur Gründung der Europäischen Gemeinschaft (EG-Vertrag) in den Fassungen des Vertrags von Nizza (abgedruckt in: Amtsblatt der EU, Nr. C 321 vom 29. Dezember 2006) und im Vergleich dazu in EUV und AEUV ergibt, dass sich die Anzahl der Nennungen von 28 auf 46 fast verdoppelt hat. Dies ist nur ein kleines Indiz für die Einschätzung.

6 Europäischer Konvent: Entwurf. Vertrag über eine Verfassung für Europa. Vom Europäischen Konvent im Konsensverfahren angenommen am 13. Juni und 10. Juli 2003, CONV 850/03.

7 Vgl. Peter Becker/Olaf Leiße: Die Zukunft Europas. Der Konvent zur Zukunft der Europäischen Union, Wiesbaden 2005 . 
Eine Analyse der oben genannten vier Elemente im Lissabonner Vertrag soll zeigen, inwieweit die Einschätzung zu seiner Bürgernähe zutreffend ist.

\section{Der Vertrag von Lissabon - ein Vertrag für die Bürger?}

Am 13. Dezember 2007 unterzeichneten die europäischen Staats- und Regierungschefs den Vertrag von Lissabon und beendeten damit mehrjährige Verhandlungen über die Reform der Europäischen Union. Der Vertrag von Lissabon enthält - nach dem gewählten Definitionsraster - folgende Verbesserungen, Weiterentwicklungen oder Neuerungen.

\section{Die Europäische Union als Wertegemeinschaft}

Der Vertrag von Lissabon nennt und bekräftigt die Werte und Ziele, auf denen die Europäische Union aufbaut. Er steht für ein Europa der Freiheit und Sicherheit und sieht neue Instrumente der Solidarität ${ }^{8}$ vor. Jeder Mitgliedstaat der Europäischen Union muss die Grundwerte achten. ${ }^{9}$ Explizit genannt werden: Menschenwürde, Freiheit, Demokratie, Gleichheit, Rechtsstaatlichkeit und der Schutz von Minderheiten. Es werden die sozialen Belange der Bürger den wirtschaftlichen Zielen der Europäischen Union gleichgestellt. Damit ist ein weiterer Schritt zur Formierung einer politischen Union eingeleitet.

Die Erfahrung terroristischer Anschläge in der Welt und Naturkatastrophen führten dazu, dass das Solidaritätsversprechen der Mitgliedstaaten untereinander konkretisiert wird. So sieht der Vertrag von Lissabon vor, dass die Europäische Union und ihre Mitgliedstaaten gemeinsam und solidarisch handeln, wenn eine der Vertragsparteien Opfer eines terroristischen Anschlags oder einer Naturkatastrophe beziehungsweise einer vom Menschen verursachten Katastrophe wird. ${ }^{10}$ Für den Unionsbürger führt diese Solidarität zu einem erhöhten $\mathrm{Ma}$ an Sicherheit - sowohl bei Vorkehrungen zur Verhinderung wie im Falle der Notwendigkeit der Hilfestellung bei einer Katastrophe.

Die Europäische Union erhält mehr Kompetenzen in den Bereichen Freiheit ${ }^{11}$, Sicherheit und Recht. ${ }^{12}$ Neue Bestimmungen zum Katastrophenschutz ${ }^{13}$, zur humanitären Hilfe ${ }^{14}$ und zur öffentlichen Gesundheit ${ }^{15}$ zielen ebenfalls darauf ab, die Europäische Union im Falle von Anschlägen auf die Sicherheit europäischer Bürger noch handlungsfähiger zu machen. Die Gewährleistung der Versorgung mit Energie ${ }^{16}$ sichert ein weiteres Feld grundlegender Bedürfnisse ab. Für die Bürger wird damit ein höheres Schutzniveau ermöglicht, in dem grenzüberschreitende Probleme mit adäquaten Mitteln bekämpft oder gelöst werden können. ${ }^{17}$

8 Das Wort wird 18-mal in EUV und AEUV verwandt, in den gemäß dem Vertrag von Nizza konsolidierten Fassungen nur sechsmal.

9 Vgl. Art. 2 EUV sowie Präambel EUV.

10 Vgl. Art. 222 AEUV.

11 Die Ausdehnung des Schengen Raumes auf das Nicht-EU-Mitglied Schweiz ist sicher ein Freiheitsgewinn für die Bürger, die jetzt darüber hinaus den grenzfreien Verkehr zwischen 22 Mitgliedstaaten der Europäischen Union sowie Norwegen und Island nutzen können.

12 So heißt es beispielsweise in Art. 81 AEUV, dass die Union eine justizielle Zusammenarbeit ,in Zivilsachen mit grenzüberschreitenden Bezug, die auf dem Grundsatz der gegenseitigen Anerkennung gerichtlicher und außergerichtlicher Entscheidungen beruht", entwickelt.

13 Vgl. Art. 196 AEUV.

14 Vgl. Art. 214 AEUV.

15 Vgl. Art. 168 AEUV.

16 Vgl. Art. 194 AEUV.

17 In den regelmäßigen Eurobarometer-Umfragen werden von den befragten Unionsbürgern die Politikfelder Terrorismus, Klimawandel, Verbraucherschutz und Gesundheit als zukünftige Prioritäten der Arbeit des Europäischen Parlaments benannt. 
Es werden immer wieder Befürchtungen laut, dass es in einer erweiterten Union zum Verlust der nationalen Identität kommt und dass sich zentralisierende Tendenzen durchsetzen werden. Um dem entgegen zu wirken heißt es an prominenter Stelle: „Die Union achtet die Gleichheit der Mitgliedstaaten vor den Verträgen und ihre jeweilige nationale Identität, die in ihren grundlegenden politischen und verfassungsmäßigen Strukturen einschließlich der regionalen und lokalen Selbstverwaltung zum Ausdruck kommt. "18 Diese Garantie trägt dem Bedürfnis der Unionsbürger Rechnung, ihre Identität - ob national, regional oder lokal verortet - nicht in einem größeren, diffusen Europabewusstsein aufgehen zu sehen. Dies widerspricht nicht der Möglichkeit zur Ausbildung einer europäischen Identität; nur ist diese eine von mehreren Optionen und soll und darf nicht die anderen ersetzen.

Zum Ausdruck kommt dieser Anspruch des Lissabonner Vertrages auch bei der Regelung, wann die Europäische Union tätig sein darf. Der Grundsatz der begrenzten Einzelermächtigung wird explizit im Lissabonner Vertrag festgeschrieben. ${ }^{19}$ Die Europäische Union wird nur im Rahmen der ihr von den Mitgliedstaaten übertragenen Zuständigkeiten tätig. Die Europäische Union darf darüber hinaus nur tätig werden, sofern und soweit beispielsweise in den Bereichen Binnenmarkt, Verbraucherschutz, Energie und innere Sicherheit die politischen Ziele auf Unionsebene besser verwirklicht werden können. Die Union muss detailliert nachweisen, dass 27 einzelstaatliche Maßnahmen einen grenzüberschreitenden Handel oder eine grenzüberschreitende Kriminalitätsbekämpfung eher verhindern als fördern. Es bleiben den Mitgliedstaaten dann immer noch Möglichkeiten gegen eine europäische Maßnahme Einspruch zu erheben.

Das mehrstufige Verfahren zur Kontrolle der Subsidiarität wird in einem Protokoll detailliert geregelt. ${ }^{20}$ Es sieht vor, dass die nationalen Parlamente schon im Stadium des Entwurfes eines Rechtsaktes diesen überprüfen und entsprechende Einwände formulieren können (Frühwarnsystem). Sie können am Ende des Rechtssetzungsverfahrens auch über die Regierungen vor dem Europäischen Gerichtshof gegen den Rechtsakt Klage erheben. Über die nationalen Parlamente hat der Bürger - neben dem Europäischen Parlament - indirekt die Möglichkeit Einfluss auf europapolitische Entscheidungen zu nehmen. Voraussetzung ist allerdings, dass er dafür oder dagegen eine entsprechende politische Mehrheit oder Öffentlichkeit mobilisieren kann. ${ }^{21}$ Die Medien spielen in diesem Zusammenhang eine große Rolle. Das Internet wird in seiner Möglichkeit, europa-, wenn nicht sogar weltweite, Meinungsbildung herzustellen hierbei in Zukunft ein immer wichtigeres Medium. ${ }^{22}$

Der Lissabonner Vertrag sieht erstmals die Möglichkeit der Rückverlagerung von Kompetenzen an die Mitgliedstaaten vor. ${ }^{23}$ Auf diese Weise begegnen die vertragsschließenden Staaten damit dem Unbehagen nicht nur der Bürger, die Europäische Union sei eine alle gesetzten Grenzen ausschöpfende beziehungsweise sogar überschreitende Organisation, die einmal auf sie übertragene Aufgaben - auch nach Erfüllung - nicht mehr aufzugeben bereit sei.

18 Vgl. Art. 4 Abs. 2 EUV.

19 Vgl. Art. 5 EUV.

20 Vgl. Protokoll (Nr. 2) über die Anwendung der Grundsätze der Subsidiarität und der Verhältnismäßigkeit, in: Amtsblatt der EU, Nr. C 115 vom 9. Mai 2008, S. 206-209.

21 Die Verhinderung von europäischen Rechtsakten erfordert einen engeren Zeitraum als den, den die alle vier oder fünf Jahre stattfindenden Wahlen zu Parlamenten ermöglichen.

22 Die dahinter stehende demokratietheoretische und -praktische Frage der Entgrenzung von Verantwortung und die Folgen für die Loyalität zu staatlichen, europäischen oder internationalen Organisationen sowie einem Gemeinwesen soll hier nicht weiter beleuchtet werden.

23 Vgl. Art 48 EUV, wo es heißt: „,Die Regierung jedes Mitgliedstaats, das Europäische Parlament oder die Kommission kann dem Rat Entwürfe zur Änderung der Verträge vorlegen. Diese Entwürfe können unter anderem eine Ausdehnung oder Verringerung der der Union in den Verträgen übertragenen Zuständigkeiten zum Ziel haben". 


\section{Rechte und Pflichten der Bürger}

Der Vertrag von Lissabon baut auf bestehende Rechte auf und führt neue Rechte für die Bürger Europas ein. Insbesondere garantiert er die Freiheiten und Grundsätze, die in der Charta der Grundrechte der Europäischen Union ${ }^{24}$ verankert sind und die sogar Rechte über die Europäische Menschenrechtskonvention ${ }^{25}$ hinaus garantiert. ${ }^{26}$ Er verleiht den Bestimmungen der Charta der Grundrechte Rechtsverbindlichkeit, auch wenn sie nicht in das Vertragswerk aufgenommen wurde, sondern sich nur ein textlicher Verweis im Vertrag befin$\operatorname{det}^{27}$ Die Charta stellt ein Kondensat aus den Verfassungen der Mitgliedstaaten, der Europäischen Menschenrechtskonvention und der Europäischen Sozialcharta ${ }^{28}$ dar. Die Bürgerinnen und Bürger werden bei der Anwendung europäischen Rechts durch die Charta und ihre gerichtliche Durchsetzung vor Verletzungen ihrer Grundrechte geschützt. „Mit der Charta der Europäischen Bürgerrechte wird der weltweit umfassendste und modernste Katalog von Rechten und Freiheiten in dem neuen Europa-Vertrag verankert. Zum ersten Mal stehen in einem Grundrechtskatalog die sozialen und wirtschaftlichen Rechte der Menschen gleichberechtigt neben den klassischen Freiheitsrechten. “29

Die Union achtet in ihrem gesamten Handeln den Grundsatz der Gleichheit ihrer Bürger. ${ }^{30} \mathrm{Im}$ Vertrag von Nizza ging es noch darum, Ungleichheiten zwischen Bürgern abzubauen und Chancengleichheit für Bürger zu ermöglichen. Der Lissabonner Vertrag geht einen Schritt weiter und formuliert eine Selbstverpflichtung der Europäischen Union, allen Bürgern ,ein gleiches Maß an Aufmerksamkeit“ zuteil werden zu lassen. Damit ist der Bürger nicht nur Objekt politischen Handelns der Europäischen Union, sondern es wird eine Beziehung zwischen den Bürgern und den Organen und anderen Gremien der Europäischen Union konstituiert.

Der Vertrag von Lissabon beinhaltet einen Ausbau des Zivil- und Personenstandsrechts bei Sachverhalten mit grenzüberschreitendem Bezug. ${ }^{31}$ So können zum Beispiel EheschlieBungen und -trennungen innerhalb der Europäischen Union schneller geregelt und die Rechte der Kinder in solchen Fällen gestärkt oder Ermittlungen und die Durchsetzung von gerichtlich erworbenen Titeln vereinfacht werden. ${ }^{32}$ Bisher mussten betroffene Bürger oft langwierige und bürokratische Hürden überwinden, um in Konfliktfällen unter Beteiligung von Personen in mehreren Mitgliedstaaten zu ihrem Recht zu kommen. Für den Bürger

24 Die Charta wurde in einem Konvent unter der Leitung des ehemaligen Bundespräsidenten Roman Herzog erarbeitet und wurde schon auf dem Europäischen Gipfel in Nizza (Dezember 2000) von den Staats- und Regierungschefs feierlich angenommen, erhielt aber keine Rechtsverbindlichkeit. Charta der Grundrechte der Europäischen Union, in: Amtsblatt der EU, Nr. C 303 vom 14. Dezember 2007, S. 1-16.

25 Konvention zum Schutze der Menschenrechte und Grundfreiheiten vom 4. November 1950 (BGB1. 1952 II, S. 686) zuletzt geändert durch das Protokoll Nr. 14 vom 13. Mai 2004 (BGB1. 2006 II, S. 138).

26 Vgl. Art. 6 EUV.

27 Allerdings haben sich Großbritannien und Polen eine Ausnahme ausgehandelt, sodass die Grundrechtecharta vor britischen und polnischen Gerichten keinen Rechtsschutz gewähren wird. Vgl. Protokoll (Nr. 30) über die Anwendung der Charta der Grundrechte der Europäischen Union auf Polen und das Vereinigte Königreich, in: Amtsblatt der EU, Nr. C 115 vom 9. Mai 2008, S. 313-314; 61. Erklärung der Republik Polen zur Charta der Grundrechte der Europäischen Union und 62. Erklärung der Republik Polen zu dem Protokoll über die Anwendung der Charta der Grundrechte der Europäischen Union auf Polen und das Vereinigte Königreich, beide in: Amtsblatt der EU, Nr. C 115 vom 9. Mai 2008, S. 358.

28 Europäische Sozialcharta vom 18. Oktober 1961 (BGB1. 1964 II, S. 1262) zuletzt geändert durch Änderungsbekanntmachung zur Europäischen Sozialcharta vom 3. September 2001 (BGB1. 2001 II, S. 970).

29 Vgl. Jo Leinen: Die Gewinner des neuen Europa-Vertrages, in: Friedrich-Ebert-Stiftung (Hrsg.): Internationale Politikanalyse, Bonn/Berlin 2008, S. 1.

30 Vgl. Art. 9 EUV.

31 Vgl. Art. 81 AEUV.

32 Vgl. Leinen: Die Gewinner des neuen Europa-Vertrages, 2008, S. 2. 
schließt sich hier die Lücke zwischen der durch die EU-Binnenmarkt-Freiheiten garantierten Freizügigkeit von Personen und der Möglichkeit grenzüberschreitend Ansprüche und Forderungen durchsetzen zu können.

Auch hat jede Person durch den Lissabonner Vertrag das Recht auf Schutz der sie betreffenden personenbezogenen Daten. ${ }^{33}$ Dieses Persönlichkeitsrecht steht in vielen Mitgliedstaaten in der Diskussion. Das Spannungsverhältnis zwischen Terror- und Kriminalitätsbekämpfung und der Wahrung von Freiheitsrechten erfordert eine Güterabwägung, die jetzt auch durch die Europäische Union abgesichert werden könnte.

Darüber hinaus wird explizit festgestellt, dass alle Bürger das Recht haben, am demokratischen Leben der Union teilzunehmen. ${ }^{34}$ Es wird zwar nicht näher ausgeführt, was konkret damit gemeint ist. Der anschließende Satz, welcher bürgernahe und offene Entscheidungen benennt, legt nahe, dass sich die Beteiligung vor allem auf die Mitwirkung der Bürger bei der Wahrnehmung ihrer demokratischen Rechte (Wahlen) und die von der Europäischen Union sicherzustellende Transparenz und Nachvollziehbarkeit ihres Handelns bezieht.

Bei der Beratung und Formulierung von Sachverhalten kann der Rat Vertreter der Zivilgesellschaft einbinden, wenn diese von der Tätigkeit der Union betroffen sind. ${ }^{35}$ Der Rat eröffnet sich die Chance, auf den Sachverstand kundiger Bürger zurückzugreifen und damit ihre Belange in die Überlegungen mit einzubeziehen. Dies funktioniert allerdings nur, wenn die betroffenen Bürger wiederum bereit sind, ihre Position bürgergesellschaftlich so zu organisieren, dass sie keine segmentierte Individualansicht widerspiegelt, sondern Interessen größerer Gruppen artikulationsfähig macht.

Interessanterweise steht dem Ausbau der Rechte des Unionsbürgers keine Einführung oder eine Ausweitung seiner Pflichten gegenüber. Im Rahmen des Europäischen Konvents wurde noch das Thema EU-Steuer (kontrovers) diskutiert; es fand aber keinen Eingang in den Lissabonner Vertrag.

\section{Demokratisierung Europas}

Das erste Mal wird im europäischen Vertragswerk der Grundsatz der ,repräsentativen Demo-

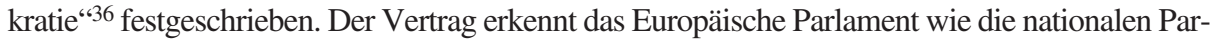
lamente als die beiden Quellen demokratischer Legitimation für das Handeln der Europäischen Union an. Sie werden direkt von den Bürgern gewählt. Es wird auch ausdrücklich klargestellt, dass sowohl die Staats- und Regierungschefs als auch die Regierungsmitglieder den Bürgern gegenüber für ihr Handeln im Rahmen der Europäischen Union rechenschaftspflichtig sind. ${ }^{37}$

Im Europäischen Parlament sind die Bürger auf Unionsebene unmittelbar vertreten. Die Kompetenzen des direkt gewählten Europäischen Parlaments in Bezug auf die Gesetzgebung, den Haushalt und internationale Übereinkommen werden erweitert. Im Vertrag wird die legislative und budgetäre Gleichberechtigung von Europäischem Parlament und Rat zum allgemeinen Rechtsgrundsatz. ${ }^{38}$ Durch die Ausdehnung des Mitentscheidungsverfahrens bei der Beschlussfassung wird zwischen dem Europäischen Parlament und dem Rat bei einem erheblichen Teil der EU-Rechtsvorschriften Gleichberechtigung hergestellt (in 95 Prozent der Fälle). ${ }^{39}$

33 Vgl. Art. 39 EUV.

34 Vgl. Art. 10 Abs. 3 EUV.

35 Vgl. Art. 302 AEUV.

36 Art. 10 Abs. 1 EUV.

37 Vgl. Art. 10 EUV.

38 Vgl. Art. 14 EUV.

39 Prozentangabe laut Elmar Brok/Martin Selmayr: Der „Vertrag der Parlamente“ als Gefahr für die Demokratie? $\mathrm{Zu}$ den offensichtlich unbegründeten Verfassungsklagen gegen den Vertrag von Lissabon, in: integration 3/2008, S. 217-234, hier S. 228. Siehe auch Art. 294 AEUV. 
Das Europäische Parlament tagte schon immer öffentlich; dies muss nun auch der Rat tun, wenn er über Gesetzgebungsakte berät oder abstimmt. ${ }^{40}$ Der Rat hat deshalb in seiner Geschäftsordnung geregelt, dass bei allen diesen Verfahren in der Mitentscheidung mit dem Europäischen Parlament eine audiovisuelle Übertragung sowie ein Video-Stream im Internet die Öffentlichkeit sicherstellen soll. Dies soll auch für den interessierten Bürger die Einsicht in europapolitische Entscheidungsgänge, Standpunkte und Interessen ermöglichen. Realistischer Weise wird diese Gelegenheit aber nur ein verschwindend geringer Prozentsatz der europäischen Bürger wahrnehmen. Zumindest aber können Journalisten diese Informationsquelle nutzen und ihrem Auftrag gemäß für eine größere Verbreitung sorgen.

Die Parlamente der Mitgliedstaaten werden das erste Mal im europäischen Vertragswerk als ,Akteure' des europapolitischen Entscheidungsganges genannt. ${ }^{41}$ Sie tragen zum reibungslosen Funktionieren der Union bei und erhalten direkte Mitwirkungsrechte im europäischen Entscheidungsprozess - und zwar jede Kammer. Für Deutschland heißt das, dass dies sowohl für den Bundestag wie den Bundesrat gleichermaßen gilt. ${ }^{42}$ In Zukunft sollen die nationalen Parlamente noch stärker darauf achten, dass die Europäische Union nur dann tätig wird, wenn auf Ebene der Europäischen Union bessere Ergebnisse erzielt werden können. Die Einhaltung dieses ,Subsidiaritätsprinzips' wird mithilfe einer neu geschaffenen Regelung verstärkt kontrolliert. Die nationalen Parlamente werden durch den Vertrag von Lissabon von ,nur mittelbar beteiligten, eher fernen Zuschauern des Unionsgeschehens zu unmittelbaren, mit eigenen justitiablen Mitwirkungsrechten ausgestatteten Akteuren des EUEntscheidungsprozesses" “. ${ }^{43}$

Neu vorgesehen ist auch die Möglichkeit der direkten Beteiligung von Bürgern an europäischer Politikgestaltung. Mittels eines Bürgerbegehrens werden eine Million Bürger aus verschiedenen Mitgliedstaaten die Möglichkeit haben, die Kommission aufzufordern, neue politische Vorschläge zu unterbreiten. ${ }^{44}$ Mit diesem Instrument wird dem vielfachen Wunsch - auch auf nationaler Ebene - Rechnung getragen, plebiszitäre Elemente in die repräsentative Demokratie einzufügen, die weiterhin das Leitbild bleibt.

Der Vertrag von Lissabon sieht erstmals die Möglichkeit zum Austritt eines Mitgliedstaates aus der Union vor. ${ }^{45}$ Auch wenn ein Austritt faktisch auch ohne diese Klausel im Vertrag möglich ist $t^{46}$ - denn kein Staat kann letztlich gegen seinen Willen in einem Vertragsverbund gehalten werden -, so schafft die explizite Erwähnung im Vertragstext Klarheit und wirkt dem Gefühl entgegen, sich mit der EU-Mitgliedschaft in eine Abhängigkeit gegen die Interessen des eigenen Staates begeben zu haben. Jeder austrittswillige Staat wird die Kosten eines solchen Schrittes trotzdem sehr wohl abwägen müssen, ebenso wie die Europäische Union.

Neben dem Argument der ,reinen Lehre` der Vertragskunst wird dem Bürger mit der ausdrücklichen Nennung der Austrittsmöglichkeit die Angst genommen, die Mitgliedschaft des eigenen Landes in der Europäischen Union enthalte einen Automatismus hin zu einer immer

40 Vgl. Art. 15 AEUV.

41 Vgl. Art. 12 EUV.

42 Vgl. Gesetzentwurf der Fraktionen CDU/CSU, SPD und Bündnis 90/Die Grünen: Entwurf eines Gesetzes über die Ausweitung und Stärkung der Rechte des Bundestages und des Bundesrates in Angelegenheiten der Europäischen Union, in: Bundestagsdrucksache 16/8489 vom 10. März 2008.

43 Brok/Selmayr: Der „Vertrag der Parlamente“ als Gefahr für die Demokratie?, 2008, S. 228.

44 Vgl. Art. 11 Abs. 4 EUV.

45 Vgl. Art. 50 EUV.

46 Es sei nur daran erinnert, dass beispielsweise Grönland nach der Erlangung seiner Autonomie 1985 faktisch aus der Europäischen Gemeinschaft ausgetreten ist. Vgl. Grönland-Vertrag, in: Amtsblatt der EG, Nr. L 29 vom 1. Februar 1985. 
weiteren Integration ohne Rückkehrmöglichkeit. Fakt ist allerdings, dass je weiter die Integration fortschreitet, die Wahrscheinlichkeit des Austritts eines Landes schwindet. Dies gilt besonders für die Staaten der Eurogruppe.

\section{Transparenz und Effizienz politischen Handelns}

Die Zweiteilung des Lissabonner Vertrages schafft mehr Transparenz für den Bürger: Im EUV sind die wesentlichen Grundsätze, die Bestimmungen über die Organe, die verstärkte Zusammenarbeit und allgemeine Bestimmungen über das auswärtige Handeln der Europäischen Union gebündelt. Er enthält die wichtigsten Regelungen - der Bürger kann sich mittels seiner einen guten Überblick über die Ziele und Zuständigkeiten der Europäischen Union verschaffen. Es ist nicht zu verschweigen, dass auch dieser Vertragsteil geprägt ist durch eine juristisch-diplomatische Sprache, ${ }^{47}$ aber trotz allem ist es ein Fortschritt, in 55 Artikeln das Grundgerüst der Europäischen Union zusammenzufassen.

Der zweite Teil ist der AEUV. Er verkörpert im Wesentlichen den , alten', an die Neuerungen im Lissabonner Vertrag angepassten EG-Vertrag: Die Funktionsweise der Organe, die internen Politiken, das auswärtige Handeln der Europäischen Union, die Finanzvorschriften, das Gesetzgebungsverfahren werden in 357 Artikeln dargelegt. ${ }^{48}$

Mit der eindeutigen Zuordnung der Zuständigkeiten wird die Beziehung zwischen den Mitgliedstaaten und der Europäischen Union klarer. Die politischen Verantwortlichkeiten werden für den Bürger besser durchschaubar. ${ }^{49}$ Der Vertrag von Lissabon schafft ein effizienteres Europa mit vereinfachten Arbeitsmethoden und Abstimmungsregeln, Institutionen, angepasst an 27 Mitgliedstaaten, und mit erhöhter Handlungsfähigkeit in den Schwerpunkttätigkeitsbereichen der Europäischen Union. Nicht gelungen ist es, die Rechtsakte der Europäischen Union den nationalen Bezeichnungen anzupassen und damit verständlicher zu machen: Auch künftig wird es immer noch Verordnungen, Richtlinien und Beschlüsse ${ }^{50}$ geben - wie im Entwurf eines Vertrags über eine Verfassung für Europa vorgesehen terminologisch von Gesetzen zu sprechen, ließ sich hingegen nicht realisieren.

Das Mitentscheidungsverfahren wird zum Regelfall. ${ }^{51}$ Die Beschlussfassung mit qualifizierter Mehrheit im Rat wird auf neue Politikbereiche ausgedehnt, um so eine schnellere und effizientere Entscheidungsfindung zu begünstigen. ${ }^{52}$ Ab 2014 wird die qualifizierte Mehrheit nach der doppelten Mehrheit von Mitgliedstaaten und Bevölkerung berechnet und ist damit Ausdruck der doppelten Legitimität der Europäischen Union. Eine doppelte Mehrheit ist dann erreicht, wenn 55 Prozent der Mitgliedstaaten, die gemeinsam mindestens 65 Prozent der europäischen Bevölkerung auf sich vereinen, zustimmen. ${ }^{53}$ Mit der obligatorischen Einführung des ,Bürger-Quorums', welches im Vertrag von Nizza nur auf Verlangen mindestens eines Mitgliedstaates erhoben werden konnte, wird dem durch die Regierungen re-

47 Auch das deutsche Grundgesetz stellt nicht in allen Passagen eine einfache Lektüre dar.

48 Zum gesamten Europa-Vertragspaket gehören darüber hinaus noch 37 Protokolle, zwei Anhänge und 65 Erklärungen.

49 Vgl. Art. 5 EUV.

50 Vgl. Art. 288 AEUV.

51 Vgl. Art. 289 AEUV wo es heißt: „Das ordentliche Gesetzgebungsverfahren besteht in der gemeinsamen Annahme [...] durch das Europäische Parlament und den Rat[...]". Ausnahmen gibt es unter anderen in den Bereichen der Außen- und Sicherheitspolitik, der polizeilichen und justiziellen Zusammenarbeit und des geistigen Eigentums.

52 Zum Beispiel in den Feldern: Polizeiliche und Justizielle Zusammenarbeit, Gemeinsame Verkehrspolitik, Asyl, Aufnahme und Ausübung selbstständiger Tätigkeiten, Kontrolle an Außengrenzen sowie diplomatischer und konsularischer Schutz.

53 Vgl. Art. 16 EUV. 
präsentierten Willen der Bevölkerung Rechnung getragen und sichergestellt, dass eine Entscheidung nicht gegen die Mehrheit der Bürger gefällt werden kann.

Mit dem Vertrag von Lissabon wird erstmals ein Präsident des Europäischen Rates von eben den Mitgliedern dieses Organs gewählt. Seine Amtszeit beträgt zweieinhalb Jahre. ${ }^{54}$ Dieses ,gewählte' Oberhaupt der Europäischen Union kann den europäischen Bürgern ein größeres Maß an Identifikation ermöglichen. Bei der Wahl des Präsidenten der Kommission durch das Europäische Parlament ist das Ergebnis der Europawahl zu berücksichtigen. ${ }^{55}$ Auch hiermit wird dem ,Bürgerwillen“ stärker Rechnung getragen, denn der Kommissionspräsident soll der politischen Richtung angehören, die bei den Wahlen die Mehrheit für sich gewinnen konnte. Die europäischen Parteien können ihre öffentliche Profilierung durch die Aufstellung von Spitzenkandidaten vorantreiben. Die Bürger erhalten die Chance, zwischen Personen und die durch sie repräsentierten Parteiprogramme zu wählen. Wie in Deutschland wählt der Bürger Abgeordnete für das Parlament in dem Wissen, dass der Spitzenkandidat der jeweiligen Partei als Kanzlerkandidat zur Verfügung steht.

Durch das Herausstellen von Kandidaten, die in der Öffentlichkeit Bekanntheit erlangen sollen, zieht die Personalisierung in die europäische Politik ein. In der heutigen vernetzten Medienwelt ist mittels geschickter Vermarktung von Politikern durch die sie nominierenden Parteien ein europaweiter Effekt möglich. Interesse bis zur Bindung des Bürgers an Persönlichkeiten soll durch solche Personalisierungen erreicht werden. Die Erfahrungen auf nationaler Ebene zeigen aber, dass die einer Person entgegengebrachte Loyalität nicht automatisch auf die durch sie repräsentierte Institution übertragen wird. Die Loyalität und diffuse Zustimmung zu den (Staats-)Organen ist aber eine der Voraussetzungen für eine funktionierende Demokratie.

Auch darf nicht übersehen werden, dass es neben dem gewählten Präsidenten des Europäischen Rates einen (vom Europäischen Parlament gewählten) Kommissionspräsidenten und einen Präsidenten des Europäischen Parlaments gibt. Die sprachlichen Doppelungen bezüglich der Bezeichnung ,Präsident', die nicht mit den nationalen Staatsgefügen deckungsgleichen Zuständigkeiten und Rechte der Organvertreter sowie die europäische Komponente des Ganzen steigern die Notwendigkeit, Kandidaten mit Persönlichkeit und Charisma zu finden, damit wirklich Identifikation und Transparenz entstehen kann. Die Erfahrung lehrt, dass erst in zweiter Linie politische Inhalte oder Botschaften hierzu beitragen.

Auch wird immer wieder eingefordert, dass die Europäische Union außenpolitisch mit einer Stimme sprechen solle. Ein neuer Hoher Vertreter der Union für die Außen- und Sicherheitspolitik wie im Lissabonner Vertrag vorgesehen, der gleichzeitig Vizepräsident der Europäischen Kommission ist, kann den Einfluss, die Stimmigkeit und die Wahrnehmbarkeit der Außenpolitik der Europäischen Union erhöhen. ${ }^{56}$ Ob dem Bürger die Zwitterstellung des Hohen Vertreters zwischen Rat und Kommission und die damit verbundenen unterschiedlichen Loyalitäten vermittelt werden können, muss sich erst noch erweisen. ${ }^{57}$

Außerdem enthält der Lissabonner Vertrag neue Bestimmungen für die künftige Zusammensetzung des Europäischen Parlaments ${ }^{58}$ und für eine Verkleinerung der Kommission. ${ }^{59}$

54 Vgl. Art. 15 Abs. 5 EUV.

55 Vgl. Art. 17 Abs. 7 EUV.

56 Vgl. Art. 18 EUV. Ein neuer Europäischer Auswärtiger Dienst soll den Hohen Vertreter in seiner Arbeit unterstützen, vgl. dazu Art. 27 EUV.

57 Im Entwurf eines Vertrags über eine Verfassung für Europa war noch ein europäischer Außenminister vorgesehen. Dieser war im Vertrag von Lissabon nicht mehr durchsetzbar. Aus dem „Hohen Vertreter für die Gemeinsame Außen- und Sicherheitspolitik“", welcher im Vertrag von Nizza in Personalunion Generalsekretär des Rates war, wurde im Lissabonner Vertrag der „Hohe Vertreter der Union für Außen- und Sicherheitspolitik“, welcher in Personalunion Vizepräsident der Kommission werden soll.

58 Vgl. Art. 14 Abs. 2 EUV.

59 Vgl. Art. 17 Abs. 4 und 5 EUV. 
Die Anzahl der Sitze im Europäischen Parlament ist bei 750 gedeckelt; die nationalen Kontingente werden neu ermittelt, um die Repräsentativität zu verbessern. ,One man, one vote ‘ ist auch nach den neuen Quoten nicht realisiert, aber es findet eine weitere Annäherung statt und damit ein Stück mehr Gerechtigkeit bei der Aufteilung der Mandate: Die Stimmenzahl zur Erreichung eines Sitzes im Parlament wird angeglichen; das Gewicht des Votums des Bürgers, unabhängig in welchem Mitgliedstaat er wählt, nähert sich weiter an. ${ }^{60}$

Die Kommission wird nach den Regelungen des Lissabonner Vertrages bis 2014 auf je einen Kommissar pro Mitgliedstaat verkleinert; ab 2014 soll die Anzahl der Kommissionsmitglieder zwei Dritteln der Zahl der Mitgliedstaaten entsprechen. Diese der Effizienz geschuldete Maßnahme wird vom Bürger eher als Verlust nationaler Einflussmöglichkeit auf europapolitische Entscheidungen empfunden. Auch die Regierungsvertreter der Mitgliedstaaten - und hier vor allem die der kleineren - sehen diese Gefahr. Über dieses Spannungsverhältnis zwischen der Effizienz von politischem Handeln und der (legitimen) Vertretung nationaler Interessen wurde in den letzten Jahren immer wieder kontrovers diskutiert - sowohl in der Politik wie in der Wissenschaft. Auf der Sitzung des Europäischen Rates im Dezember 2008 wurde festgelegt, dass bei Inkrafttreten des Lissabonner Vertrages ein Beschluss gefasst wird, wonach weiterhin ein Staatsangehöriger jedes Mitgliedslandes der Kommission angehören wird. ${ }^{61}$ Damit hat der Europäische Rat in diesem Punkt noch vor Inkrafttreten des Lissabonner Vertrages eine Revision beschlossen.

$\mathrm{Zu}$ einem besseren Verständnis führt der Verzicht auf die Unterscheidung zwischen obligatorischen und nicht-obligatorischen Haushaltsmitteln. ${ }^{62}$ Die Europäische Union finanziert sich durch Eigenmittel, die nach , einem ' Haushaltsverfahren, an dem das Europäische Parlament und der Rat gleichberechtigt teilnehmen, bewirtschaftet werden.

Der Vertrag von Lissabon verbessert die Handlungsfähigkeit der Europäischen Union in politischen Bereichen, die für die heutige Europäische Union und ihre Bürger Priorität haben. Dies gilt insbesondere für die Bereiche Freiheit, Sicherheit und Recht und vor allem für die Terrorismus- und Verbrechensbekämpfung. In geringerem Maße gilt dies auch für Bereiche wie Energiepolitik, öffentliche Gesundheit, Zivilschutz, Klimawandel, Dienstleistungen von allgemeinem Interesse, Forschung, Raumfahrt, räumlicher Zusammenhalt, Handelspolitik, humanitäre Hilfe, Sport, Tourismus und administrative Zusammenarbeit.

\section{Von einem ,Europa für die Bürger' zu einem ,Europa der Bürger'}

Die Analyse des Lissabonner Vertrages hat eines deutlich werden lassen: Auch wenn mit dem Lissabonner Vertrag auf der europäischen Ebene längst noch nicht die Beteiligungsmöglichkeiten für den Bürger gegeben sind, wie auf der nationalen Ebene, so ist doch das Bemühen erkennbar, Europa mit seinen Bürgern zu versöhnen und um Interesse und Engagement für Europa zu werben. Die Tatsache, dass die Europäische Union kein Staat im landläufigen Sinne ist und die Mitgliedstaaten wesentliche Teile ihrer Souveränität nicht abgegeben haben sowie dass sich die Völker Europas nicht als ,ein“ europäisches Volk verstehen, bildet allerdings auf absehbare Zeit eine Grenze für die Entwicklung der europäischen Demokratie nach nationalen Blaupausen.

60 Vgl. Art. 14 EUV: Die Bürger sind im Europäischen Parlament degressiv proportional mindestens jedoch mit sechs Mitgliedern je Mitgliedstaat vertreten; kein Mitgliedstaat erhält mehr als 96 Sitze.

61 Vgl. Rat der Europäischen Union: Tagung des Europäisches Rates vom 11./12. Dezember 2008 in Brüssel. Schlussfolgerungen des Vorsitzes, Dok. 17271/1/08 REV 1.

62 Vgl. Art. 311 AEUV. 
Europapolitik soll aber nicht nur Objekt von Betrachtung und Nutzung sein, sondern den Bürger aktivieren sich dieser Ebene politischer Entscheidungen auch zu bedienen und soweit es möglich ist mitzuwirken am Zustandekommen dergleichen. Das setzt voraus, dass die Bürger von den Instrumenten Kenntnis haben, aber auch willens und bereit sind, diese zu nutzen. ${ }^{63}$

Die Identifikation des Bürgers mit der europäischen Idee und der europäischen Einigung wird nicht gerade erleichtert, wenn die Europäische Union beziehungsweise ihre Mitgliedstaaten im Gegensatz zum Verfassungsvertragsentwurf im Vertrag von Lissabon auf Symbole wie Flagge, Hymne und Europatag verzichten. In der Praxis wird sich am Gebrauch der Symbole zwar nichts ändern, da diese auch bisher verwendet wurden, ohne dass es dafür eine ausdrückliche vertragliche Grundlage gab. Aber durch dieses ,Hin und Her" wurde in der Öffentlichkeit erst so recht deutlich, dass der Europäischen Union die Kraft, zu ihren Symbolen zu stehen, fehlt und die Mitgliedstaaten hier eher eine Bedrohung ihrer eigenen Identität sehen.

Die Europäische Union hat den Bereich der ,Bürgernähe' in den Vertragsreformen immer weiter ausgebaut (Ombudsmann, Petitionsausschuss, Direktwahl des Europäischen Parlaments, Unionsbürgerschaft usw.); hierin ist viel Anstrengung investiert worden. Auch an der Steigerung der ,Bürgerfreundlichkeit‘ (Transparenz, Personalisierungen, Zugang zu Informationen usw.) ist in den letzten Jahren gearbeitet worden.

Das Kriterium ,bürgertauglich“ ist eher als ambivalent zu bewerten: Auf der einen Seite gibt es Ansätze dazu (Bürgerbegehren, andere Elemente direkter und indirekter Einflussnahme usw.), auf der anderen Seite stellt sich die Frage, inwiefern ein System wie die Europäische Union dies grundsätzlich leisten kann. Ist es der vierten politischen Ebene - nach kommunal, regional, national - nicht immanent, dass die ,Bürgertauglichkeit' nur sehr begrenzt gewährleistet sein kann? Die Einflussnahme auf europäische Entscheidungsprozesse bedarf eines hohen Grades an grenzüberschreitender Organisations- und Mobilisierungskraft und fordert auch entsprechend, qualifizierte“ Bürger. Über das Internet lässt sich eine europäische Öffentlichkeit herstellen, die bisher in der Medienlandschaft immer wieder angemahnt wurde - wobei die Gefahr des Fehlens von Legitimität besteht und die Frage der letztendlichen Verantwortlichkeit unbeantwortet bleiben muss.

Trotzdem bleibt die Verantwortung des - immer wieder vielzitierten - mündigen Bürgers mit dem ihm zur Verfügung stehenden Mitteln zum Gelingen der Demokratie - ob national oder europäisch - beizutragen. Dies kann er in Ausübung seines Wahlrechtes.

\section{Teilnahme an der Europawahl ist erste Bürgerpflicht}

Die Bedeutung der Europäischen Union in Hinblick auf die Gestaltung wesentlicher Politikfelder hat in den letzten Jahren zugenommen. Dem stehen widersprüchliche Einschätzungen der Bürgerinnen und Bürger entgegen: Auf der einen Seite erscheint ,Brüssel ${ }^{`}$ - als Synonym für die Europäische Union - fern und machtlos, auf der anderen Seite wird eine Überregulierung - Eurokratie - befürchtet, auf die kein Einfluss genommen werden kann. Auch wenn es unbestritten ist, dass die Demokratisierung Europas noch Defizite aufweist, gibt es doch eine Reihe von Ansatzpunkten für ein Mitwirken der Bürgerinnen und Bürger am europäischen Geschehen. Wie die Analyse gezeigt hat, ist der Lissabonner Vertrag ein

63 Siehe auch Bundeszentrale für politische Bildung (Hrsg.): Europäische Union, Informationen zur politischen Bildung Nr. 279, Bonn 2006; Wolfram Hilz/Volker Kronenberg/Melanie Piepenschneider (Hrsg.): Auf dem Weg zu mehr Demokratie und Bürgernähe. Europas Zukunft nach dem Lissabonner Vertrag, Sankt Augustin 2009. 
wichtiger und weitergehender Schritt zum Abbau des Demokratiedefizits und zum Aufbau einer neuen Beziehung zwischen Bürger und der europäischen Einigung.

Unabhängig davon ist es vornehmste Pflicht eines Bürgers an den Wahlen zu den nationalen Verfassungsorganen, aber auch zu den Organen der Europäischen Union teilzunehmen. Die Mitglieder des Europäischen Parlaments sind die einzigen Mandatsträger für die europäische Politikebene, die direkt von den Bürgern der Europäischen Union gewählt werden können. Die Wahlbeteiligung bei der letzten Europawahl im Jahr 2004 lag im Durchschnitt unter 50 Prozent und war die niedrigste seit der Einführung der Direktwahl 1979.64

Tabelle: Wahlbeteiligung bei den Wahlen zum Europäischen Parlament 1979 bis 2004

\begin{tabular}{|l|c|c|c|c|c|c|}
\hline & $\mathbf{1 9 7 9}$ & $\mathbf{1 9 8 4}$ & $\mathbf{1 9 8 9}$ & $\mathbf{1 9 9 4}$ & $\mathbf{1 9 9 9}$ & $\mathbf{2 0 0 4}$ \\
\hline EU & $63,0 \%$ & $61,0 \%$ & $58,5 \%$ & $56,8 \%$ & $49,8 \%$ & $45,6 \%$ \\
\hline Deutschland & $65,7 \%$ & $56,8 \%$ & $62,3 \%$ & $60,0 \%$ & $45,2 \%$ & $43,0 \%$ \\
\hline
\end{tabular}

Quelle: Entwicklung der Wahlbeteiligung bei Europawahlen 1979 bis 2004, abrufbar unter: http:// www.europarl.europa.eu/elections2004/ep-election/sites/de/results1306/turnout_ep/turnout_table.html (letzter Zugriff: 24. Februar 2009).

Die Attraktivität der Wahlen zum Europäischen Parlament scheint immer mehr abzunehmen - bei gleichzeitigem Zuwachs an Kompetenzen für das Europäische Parlament und bei steigenden Möglichkeiten der Einflussnahme der Bürger auf die Geschehnisse in der Europäischen Union. Für diese paradoxe Situation gibt es unterschiedliche Erklärungsansätze. So ist schwer zu vermitteln, worum es bei den Europawahlen geht. Die Wahlkämpfe werden nach nationalem Wahlrecht, mit nationalen Kandidaten und in nationalen Wahlkreisen meist auch noch mit nationalen Themen geführt. Es geht noch nicht - da der Lissabonner Vertrag noch nicht in Kraft ist - darum, eine europäische Regierung oder einen europäischen Präsidenten zu wählen.

Außerdem haben sich noch keine ,echten` europäischen Parteien ausgebildet, sondern es handelt sich mehr um europäische Parteienzusammenschlüsse, die eine Art Dachorganisation für die nationalen Parteien bilden. Die politisch wichtigen Entscheidungen und programmatischen Positionierungen fallen aber immer noch vor allem im Rahmen der nationalen Parteienstrukturen. Zudem unterscheiden sich die Parteien in ihren europapolitischen Positionen nur in Details; erst in den letzten Jahren, seit sich auch die Europapolitik immer stärker ausdifferenziert hat, sind Ansätze für parteipolitische Trennschärfen zu erkennen.

Auch die nationale Ebene wird (noch) nicht so recht als Austragungsort unterschiedlicher europapolitischer Positionen wahrgenommen. Durch die bisherige Intransparenz, welche politische Ebene für welche Entscheidungen die Verantwortung trägt, können die nationalen oder regionalen Abgeordneten und die Regierungen für ihre europapolitischen Entscheidungen zu wenig zur Rechenschaft gezogen werden. In Deutschland werden die Bundestagsbeziehungsweise die Landtagsabgeordneten und die Bundes- oder Länderregierungen nicht an ihrer Europapolitik gemessen.

64 Dabei sprechen die Unionsbürger in den Eurobarometer-Umfragen dem Europäischen Parlament im hohen Maße das Vertrauen aus, nach Europäischem Gerichtshof und Europäischer Zentralbank. Vgl. Europäische Kommission: Standard Eurobarometer 68. Die öffentliche Meinung in der Europäischen Union, Mai 2008, S. 93. 
Dies alles bildet Nährboden für anti-europäische Kräfte, die darauf hinarbeiten, eine vernehmbare Stimme auch im Europäischen Parlament zu werden. ${ }^{65}$ Sie werden versuchen durch eine hohe Repräsentanz im einzig direkt gewählten Organ der Europäischen Union, die Europäische Union von innen her auszuhöhlen.

\section{Mitwirken an Europa ist kein politischer Luxus}

Der Europaabgeordnete Elmar Brok plädiert dafür, den Vertrag von Lissabon als „Vertrag der Parlamente“ zu bezeichnen. Denn kein anderer Vertrag oder keine andere Vertragsänderung hat einen solchen „Beitrag zur Parlamentarisierung der europäischen Entscheidungsverfahren" geleistet. ${ }^{66}$

In den beim Bundesverfassungsgericht eingereichten Klagen beruft man sich darauf, dass der Vertrag von Lissabon die Bürger in den Entscheidungsprozessen völlig entmachte. Die Analyse hat gezeigt, dass die Bürger Europas mit diesem Vertrag mehr denn je Einflussmöglichkeiten auf die europäische Einigung haben könnten. Voraussetzung ist allerdings, dass sie ihre Rechte auch offensiv wahrnehmen. Demokratie ist eine der schwierigsten Formen zur Organisation eines Gemeinwesens: Sie hat nämlich die Wahrnehmung von Verantwortung durch Demokraten zur Voraussetzung.

Nationale Referenden werden immer wieder als Mittel empfohlen, die Bürger mit Europa zu versöhnen. Auffällig ist indes, dass ein ablehnendes Referendum wie im Juni 2008 in Irland über den Lissabonner Vertrag keine Aussage über die generell hohe Zustimmung zur europäischen Integration der Iren darstellt. Daraus lässt sich der Schluss ziehen, dass die Durchführung von Referenden politisch abgewogen werden muss, damit nicht kampagnenfähige und mobilisierungsstarke Partikularinteressen solche plebiszitären Elemente wie ein Referendum instrumentalisieren. Auf der anderen Seite hat das Referendum in Irland auch deutlich gemacht, dass sich die Zivilgesellschaft engagiert (wenn in diesem Fall auch die Nein-Sager überwogen), das heißt eine Befassung des Bürgers mit Europa stattfindet. ${ }^{67}$

Den Bürgern muss plausibel werden, dass Europa keine nutzlose technokratische Superbürokratie mit Selbstbeschäftigungscharakter ist. Dies fordert die Europäische Union, ihre Repräsentanten, die Mitgliedstaaten und ihre Akteure sowie den Bürger selbst. Eine Auseinandersetzung mit Europa und ein Mitwirken an Europa ist kein politischer Luxus, sondern Notwendigkeit für das (Über-)Leben in einer globalisierten Welt.

65 Siehe auch Sabine Riedel: Nationalismus im EU-Parlament. Parteien, Standpunkte und Gegenstrategien vor den Europawahlen 2009, SWP-Studie 37/2008.

66 Brok/Selmayr: Der „Vertrag der Parlamente“ als Gefahr für die Demokratie?, 2008, S. 217, 225.

67 Markus Gastinger/Julia Lieb/Andreas Maurer/Marie McGinley: Das Irische Nein zum Lissabonner Vertrag. Kampagnen, Abstimmungsmotive und Perspektiven des Reformvertrages, SWP-Diskussionspapier der FG 1 15/2008 und der FG 2 7/2008, vor allem S. 14-17. 